\title{
Multi-scale computational modeling of two-phased metal using GMC method
}

\author{
M. Ghorbani Moghaddam ${ }^{1}$ and A. Achuthan. ${ }^{2}$ \\ Clarkson University, Potsdam, NY, 13699, USA \\ B. A. Bednarcyk ${ }^{3}$, S. M. Arnold ${ }^{4}$ and E. J. Pineda ${ }^{5}$ \\ NASA Glenn Research Center, Cleveland, OH, 44135, USA
}

\begin{abstract}
A multi-scale computational model for determining plastic behavior in two-phased CMSX-4 Ni-based superalloys is developed on a finite element analysis (FEA) framework employing crystal plasticity constitutive model that can capture the microstructural scale stress field. The generalized method of cells (GMC) micromechanics model is used for homogenizing the local field quantities. At first, GMC as stand-alone is validated by analyzing a repeating unit cell (RUC) as a two-phased sample with $\mathbf{7 2 . 9 \%}$ volume fraction of $\gamma$-precipitate in the $\gamma$-matrix phase and comparing the results with those predicted by finite element analysis (FEA) models incorporating the same crystal plasticity constitutive model. The global stress-strain behavior and the local field quantity distributions predicted by GMC demonstrated good agreement with FEA. High computational saving, at the expense of some accuracy in the components of local tensor field quantities, was obtained with GMC. Finally, the capability of the developed multi-scale model linking FEA and GMC to solve real life sized structures is demonstrated by analyzing an engine disc component and determining the microstructural scale details of the field quantities.
\end{abstract}

\section{Introduction}

Single crystal materials such as Nickel-based super alloys (e.g. ME3) are widely used in engine blades and disks due to their exceptional strength, fracture toughness and resistance against creep and corrosion at elevated temperature and other harsh environments. Nickel-based super alloys contain two-phased composite microstructures; $\gamma$-matrix phase (predominantly an alloy of $\mathrm{Ni}, \mathrm{Cr}$ and other alloying elements with a face-centered cubic (fcc) lattice Structure) and the precipitate phase $\gamma^{\prime}$ (a coherent, ordered Ni3Al inter-metallic of LI2 crystal structure). The morphology and the distribution of the $\gamma^{\prime}$ precipitates inside $\gamma$ matrix influences the deformation, deriving excellent mechanical properties. Therefore, the volume fraction of precipitates, their mean size and spacing have a major role on the behavior of these alloys in different loading and temperature conditions. Comparing to the matrix phase, precipitates show much less nonlinear plastic behavior. As a result, the precipitates act as obstacles to the motion of dislocations in the matrix ${ }^{1-3}$.

In order to predict the microstructural plastic behavior in the two-phased Ni-based super alloys, models that can incorporate the interaction of various microstructural features, such as grain boundaries, material phases and dislocations are needed. The two key characteristics associated with plasticity are the permanent strain due to slipping of atomic layers under shear stress and the strain hardening resulting in nonlinear stress-strain behavior. In the crystal plasticity models, the dislocation motion is incorporated as permanent shear strains in the various slip systems (slip plane and slip direction) implemented with the aid of a set of state variables ${ }^{4,5}$. The strain hardening is implemented in terms of the strength of slip systems, another set of state variables. The nonlinear evolution laws are defined for the permanent shear strains and strength of slip systems, along with the equilibrium equations in an

${ }^{1} \mathrm{PhD}$ Candidate, Mechanical and Aeronatical Engineering, ghorbam@clarkson.edu.

${ }^{2}$ Assistant Professor, Mechanical and Aeronatical Engineering, aachutha@clarkson.edu.

${ }^{3}$ Materials Research Engineer, Mechanics and Life Prediction Branch, brett.a.bednarcyk@nasa.gov, AIAA Associate Fellow.

${ }^{4}$ Chief of Mechanics and Life Prediction Branch, Structures and Materials Division,, steven.m.arnold@nasa.gov, AIAA member.

${ }^{5}$ Aerospace Research Engineer, Mechanics and Life Prediction Branch, evan.j.pineda@nasa.gov, AIAA member.

1

American Institute of Aeronautics and Astronautics 
incremental and iterative approach. Several studies on the development of the crystal plasticity models with constitutive relations defining shear flow and hardening rates for Ni-based superalloys have been reported in the literature ${ }^{6-14}$. Studies focusing on the interaction of two phases by implementing constitutive models for the individual phases separately have also been reported (Refs. 6, 12 and 13).

The major challenge in implementing microstructure based plasticity models to real sized structural components is the computational cost as it requires solving governing equations at both the microstructural and macrostructural length scales. A potential solution for addressing this challenge is the careful implementation of an appropriate and efficient homogenization technique to bridge between the micro-scale and the macro-scale deformation without significantly compromising on the accuracy of the solution, especially in the critical regions of interest.

In this study, the generalized method of cells (GMC) ${ }^{15-19}$ is applied as an integrated homogenization method which allows linking the macro-scale to the microstructure while considering the individual microstructural phases explicitly. To this end, a crystal plasticity model in the form of a user material subroutine is implemented in the constituents at the micro-scale using GMC homogenization ${ }^{20,21}$. At first, the performance of the stand-alone GMC, in terms of solution accuracy and computational time, was evaluated by comparing with a finite element model that uses the same material subroutine. Finally, the multi-scale model, implemented on finite element analysis framework at macro-scale with element properties defined at micro-scale using GMC homogenization, was evaluated by analyzing a realistic engine disc component.

\section{Single-crystal Plasticity Framework}

The crystal plasticity theory is based on the assumption that any elasto-plastic deformation can be split into two multiplicative operations, a plastic deformation where material is deformed through the rearrangement of lattices but not through its own deformation, followed by an elastic deformation associated with the stretching of lattices ${ }^{22-24}$. The total deformation gradient $\boldsymbol{F}$ is then given by,

$$
F=F^{e} . F^{p}
$$

where $\boldsymbol{F}^{p}$ and $\boldsymbol{F}^{e}$ are the plastic and elastic deformation gradient, respectively.

Based on the deformation gradient definition, the total velocity gradient $\boldsymbol{L}$ is stated as

$$
\boldsymbol{L}=\dot{\boldsymbol{F}} \cdot \boldsymbol{F}^{-1}=\boldsymbol{D}+\boldsymbol{\Omega}
$$

which the symmetric stretch rate $\boldsymbol{D}$ and the anti-symmetric spin tensor $\boldsymbol{\Omega}$ can be decomposed into lattice and plastic parts $\left(()^{*},()^{p}\right)$, respectively.

$$
D=D^{*}+D^{p} \quad, \Omega=\Omega^{*}+\Omega^{p}
$$

The velocity gradient associated with the plastic deformation $\boldsymbol{L}^{p}$ is given in terms of Schmid's law as,

$$
\boldsymbol{L}^{\boldsymbol{p}}=\boldsymbol{F}^{p} \cdot \dot{\boldsymbol{F}}^{\boldsymbol{p}^{-1}}=\sum_{\alpha} \dot{\gamma}^{\alpha} \boldsymbol{m}^{\alpha} \otimes \boldsymbol{n}^{\alpha}
$$

where $\dot{\gamma}^{\alpha}$ is the rate of shear strain associated with the slipping of $\alpha$ slip system, $\boldsymbol{m}$ is the unit normal to the slip plane and $\boldsymbol{n}$ is the unit vector parallel to slip direction. The incremental formulation of plasticity theory is based on (1) the evolution of Cauchy stress on the corotational frame of reference that rotate with the crystal lattice, $\boldsymbol{J}^{*}(\boldsymbol{\sigma}),(2)$ the slipping rate $\dot{\gamma}^{(\alpha)}$ and (3) the strain hardening rate $\dot{g}^{(\alpha)}$ as given below

$$
\boldsymbol{J}^{*}(\boldsymbol{\sigma})+\boldsymbol{\sigma}\left(\boldsymbol{I}: \boldsymbol{D}^{*}\right)=\boldsymbol{C}: \boldsymbol{D}^{*},
$$

where $\boldsymbol{\sigma}$ is the Cauchy stress, $\boldsymbol{D}^{*}$ is rate of stretching associated with elastic deformation and $\boldsymbol{C}$ is the tensor of elastic moduli. The governing equation for slipping is defined as

$$
\dot{\gamma}^{(\alpha)}=\dot{a}^{(\alpha)}\left(\frac{\tau^{\alpha}}{g^{\alpha}}\right)^{n}
$$

where $\tau^{\alpha}$ is the resolved shear stress, $g^{\alpha}$ is the strength, the $\dot{a}^{(\alpha)}$ refers to the slipping rate when resolved shear stress reaches the strength. The governing equation for the strength can be modeled as

$$
\dot{g}^{(\alpha)}=\sum_{\beta} h_{\alpha \beta} \dot{\gamma}^{(\alpha)}
$$

where $h_{\alpha \beta}$ is the slip hardening moduli. The sum ranges over all activated slip systems. $h_{\alpha \alpha}$ and $h_{\alpha \beta}$ are called self and latent hardening moduli, respectively.

Hardening is defined based on Pierce ${ }^{25}$ and Asaro ${ }^{26}$ model

$$
\left.h_{\alpha \alpha}=h(\gamma)=h_{0} \sec ^{2}\left|\frac{h_{0} \gamma}{\tau_{s}-\tau_{0}}\right| \text { (no sum on } \alpha\right)
$$

Where $h_{0}$ is the initial hardening modulus, $\tau_{0}$ is the yield stress which equals the initial value of current strength $g^{(\alpha)}(0), \tau_{s}$ is the stage-I stress (or the break-through stress where large plastic flow initiates), and $\gamma$ is the Taylor cumulative shear strain on all slip systems, i.e.

$$
\gamma=\sum_{\alpha} \int_{0}^{t}\left|\dot{\gamma}^{(\alpha)}\right| d t
$$

The latent hardening modlui are given by 
Where $q$ is a constant.

$$
h_{\alpha \beta}=q h(\gamma) \quad(\alpha \neq \beta)
$$

For the numerical implementation of single crystal plasticity model a user material subroutine in the form of UMAT for Abaqus ${ }^{27}$ is used ${ }^{4,5}$.

\section{Generalized Method of Cells (GMC) framework}

GMC is a micromechanical formulation for predicting the overall thermo-inelastic behavior of the multi-scale composites. There are four steps involved in this homogenization process ${ }^{15,16}$. First the RUC should be identified (Figure 1a) and discretized. A typical RUC is consisted of $N_{\alpha} \times N_{\beta} \times N_{\gamma}$ rectangular subcells $\left(\alpha=1, \ldots, N_{\alpha} ; \beta=\right.$ $\left.1, \ldots, N_{\beta} ; \gamma=1, \ldots, N_{\gamma}\right)$ in the $x_{1}, x_{2}$ and $x_{3}$ directions, respectively, as shown in Figure $1 \mathrm{~b}$. The individual subcells have the dimensions of $\left(d_{\alpha}, h_{\beta}, l_{\gamma}\right)$ and are related to the unit cell dimensions $(d, h, l)$ by,

$$
d=\sum_{\alpha=1}^{N_{\alpha}} d_{\alpha}, \quad h=\sum_{\beta=1}^{N_{\beta}} d_{\beta}, \quad l=\sum_{\gamma=1}^{N_{\gamma}} d_{\gamma}
$$

a)

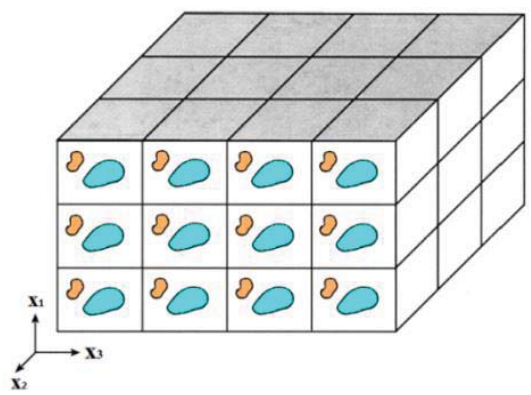

b)

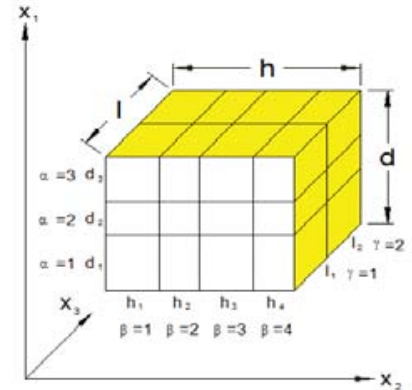

Figure 1. a) Repeating Unit Cells (RUCs) b) A sample RUC discretization consisted of $\boldsymbol{N}_{\boldsymbol{\alpha}}=\mathbf{3}, \boldsymbol{N}_{\boldsymbol{\beta}}=\mathbf{4}$ and $\boldsymbol{N}_{\gamma}=2$ subcells $^{16}$

As the second step, the relationships between the macroscopic average stresses and strains with the microscopic ones are stated. A linearly variable displacement $u_{i}^{(\alpha \beta \gamma)}(i=1,2,3)$ is considered inside each subcell. This displacement profile is consisted of pure displacement components in the center of each unit cell and the microvariables representing the linear dependence of the displacement to the local coordinates.

The average strain in the RUC is defined as

$$
\bar{\varepsilon}_{i j}=\frac{1}{d h l} \sum_{\alpha=1}^{N_{\alpha}} \sum_{\beta=1}^{N_{\beta}} \sum_{\gamma=1}^{N_{\gamma}} d_{\alpha} h_{\beta} l_{\gamma} \bar{\varepsilon}_{i j}^{(\alpha \beta \gamma)}
$$

which is based on the definition of strain tensor in each subcell $\overline{\boldsymbol{\varepsilon}}_{i j}^{(\alpha \beta \gamma)}$ as:

$$
\bar{\varepsilon}_{i j}^{(\alpha \beta \gamma)}=\frac{1}{2}\left(\partial_{i} u_{j}^{(\alpha \beta \gamma)}+\partial_{j} u_{i}^{(\alpha \beta \gamma)}\right)
$$

Considering a general constitutive equation for the elastic-viscoplastic temperature-dependent material in each subcell $(\alpha, \beta, \gamma)$, the average stress in that subcell is considered as,

$$
\bar{\sigma}_{i j}^{(\alpha \beta \gamma)}=C_{i j k l}^{(\alpha \beta \gamma)}\left(\bar{\varepsilon}_{k l}^{(\alpha \beta \gamma)}-\bar{\varepsilon}_{k l}^{I(\alpha \beta \gamma)}-\bar{\varepsilon}_{k l}^{T(\alpha \beta \gamma)}\right)
$$

where the $C_{i j k l}^{(\alpha \beta \gamma)}$ is the elastic tensor. The $\bar{\varepsilon}_{k l}^{I(\alpha \beta \gamma)}$ and $\bar{\varepsilon}_{k l}^{T(\alpha \beta \gamma)}$ are the average inelastic and thermal strain tensors, respectively, in each subcell.

Based on the volumetric summation of the average stresses in all subcells, the average stress in the entire unit-cell can be written as,

$$
\overline{\boldsymbol{\sigma}}_{i j}=\frac{1}{d h l} \sum_{\alpha=1}^{N_{\alpha}} \sum_{\beta=1}^{N_{\beta}} \sum_{\gamma=1}^{N_{\gamma}} d_{\alpha} h_{\beta} l_{\gamma} \overline{\boldsymbol{\sigma}}_{i j}^{(\alpha \boldsymbol{\beta} \gamma)}
$$

In the third step the continuity of displacement and traction is applied in an average, or integral, sense. It is considered that the RUCs are periodic and the interfaces of the subcells and the boundaries of the RUCs follow the displacement and traction continuity. Applying these conditions results in elimination of the micro-variables in the displacement definition and produces a set of continuity relationships. As a result, a set of $N_{\alpha}\left(N_{\beta}+N_{\gamma}+1\right)+$ $N_{\beta}\left(N_{\gamma}+1\right)+N_{\gamma}$ equations stems from the strain continuity relation which can be declared as:

$$
A_{G} \varepsilon_{s}=J \bar{\varepsilon}
$$


with $\boldsymbol{A}_{\boldsymbol{G}}$ and $\boldsymbol{J}$ being matrices representing the geometrical details of the subcells and those related to the unit-cell itself, respectively. The $\bar{\varepsilon}$ is the average strain in the unit-cell, while $\varepsilon_{\boldsymbol{s}}$ is defined as,

$$
\varepsilon_{s}=\left(\bar{\varepsilon}^{(111)}, \ldots, \bar{\varepsilon}^{\left(N_{\alpha}, N_{\beta}, N_{\gamma}\right)}\right)
$$

The continuity of traction, would result in

$$
A_{M}\left(\varepsilon_{s}-\varepsilon_{s}^{I}-\varepsilon_{s}^{T}\right)=0
$$

which contains $6 N_{\alpha} N_{\beta} N_{\gamma}-\left(N_{\alpha} N_{\beta}+N_{\alpha} N_{\gamma}+N_{\beta} N_{\gamma}\right)-\left(N_{\alpha}+N_{\beta}+N_{\gamma}\right)$ equations, in which $\boldsymbol{A}_{M}$ includes the information regarding the material properties in each subcell. $\varepsilon_{s}^{I}$ and $\varepsilon_{s}^{T}$ represent the inelastic and thermal strains in each subcell and are defined in a similar way as Eq. 17.

The strain and traction continuity conditions (Eqns. (16) and (17) can be combined as

where,

$$
\widetilde{A} \varepsilon_{s}-\widetilde{D}\left(\varepsilon_{s}^{I}+\varepsilon_{s}^{T}\right)=K \bar{\varepsilon}
$$

Rewriting this equation for each subcell as

$$
\widetilde{A}=\left[\begin{array}{l}
A_{M} \\
A_{G}
\end{array}\right], \quad \widetilde{D}=\left[\begin{array}{c}
A_{M} \\
0
\end{array}\right], \quad K=\left[\begin{array}{l}
0 \\
J
\end{array}\right],
$$

$$
\bar{\varepsilon}^{(\alpha \beta \gamma)}=A^{(\alpha \beta \gamma)} \bar{\varepsilon}+D^{(\alpha \beta \gamma)}\left(\varepsilon_{s}^{I}+\varepsilon_{s}^{T}\right)
$$

allows expressing the average strain in the subcell in terms of the macrostrain, and the inelastic and thermal strains by defining the concentration matrices $\boldsymbol{A}^{(\alpha \beta \gamma)}$ and $\boldsymbol{D}^{(\alpha \beta \gamma)}$.

In the fourth step, the overall macroscopic constitutive equations of the material based on the effective properties can be determined. The average stress in each subcell

$$
\bar{\sigma}=B^{*}\left(\bar{\varepsilon}-\bar{\varepsilon}^{I}-\bar{\varepsilon}^{T}\right)
$$

in which the $\boldsymbol{B}^{*}$ represents the effective elastic tensor of the composite:

$$
\boldsymbol{B}^{*}=\frac{1}{d h l} \sum_{\alpha=1}^{N_{\alpha}} \sum_{\beta=1}^{N_{\beta}} \sum_{\gamma=1}^{N_{\gamma}} d_{\alpha} h_{\beta} l_{\gamma} \boldsymbol{C}^{(\alpha \boldsymbol{\beta} \gamma)} \boldsymbol{A}^{(\alpha \beta \gamma)}
$$

The inelastic strain tensor $\overline{\boldsymbol{\varepsilon}}^{I}$ and the average thermal strain tensor $\overline{\boldsymbol{\varepsilon}}^{T}$ are also obtained in a similar fashion.

This method can be reformulated using subcell stresses rather than strains as the unknown quantities which reduces the computational cost significantly ${ }^{28}$.

\section{Proposed mutli-scale model}

The Micromechanics Analysis Code with Generalized Method of Cells (MAC/GMC), developed by NASA Glenn Research Center that predicts the effective nonlinear response of heterogeneous materials using GMC based on the known behavior of the constituent materials is used in the present study. The MAC/GMC software, like most of the commercially available FEA packages, admits user-defined constitutive models. This capability was used to incorporate the single crystal plasticity model within the code such that the behavior of the individual constituent materials could be represented with this model. The GMC is called at finite element, integration point level to represent the material response of that element using NASA's FEAMAC software ${ }^{27,20,21}$.

\section{Numerical Implementation of two-phased single crystal materials}

In this study, CMSX-4 Nickel-based super alloy which is extensively used for turbine blades is chosen. This alloy has an average precipitate size of $0.45 \mu \mathrm{m}$ and volume fraction of roughly $70 \%$ (Figure 2 ) (in this study 0.729 ). The composition of CMSX-4 for each phase is shown in Table $1^{29}$.

Table 1. Nominal composition of alloy (CMSX-4) (at \%) as given in ${ }^{29}$

\begin{tabular}{|c|c|c|c|c|c|c|c|c|c|c|}
\hline & $\mathbf{N i}$ & $\mathbf{A l}$ & $\mathrm{Ti}$ & $\mathrm{Ta}$ & $\mathbf{C r}$ & $\mathbf{C o}$ & $\mathbf{W}$ & $\mathbf{M o}$ & $\mathbf{R e}$ & Hf \\
\hline CMSX-4 & 63.1 & 12.5 & 1.3 & 2.0 & 7.6 & 10.3 & 2.0 & 0.4 & 1.0 & 0.1 \\
\hline Matrix $(\gamma)$ & 54.9 & -- & -- & -- & 20 & 18.6 & 2.7 & 0.8 & 3 & -- \\
\hline Inclusion $\left(\gamma^{\prime}\right)$ & 75 & 23 & 2 & 1 & -- & -- & -- & -- & -- & -- \\
\hline
\end{tabular}

The inclusion is considered to be totally elastic while the matrix demonstrates elasto-plastic behavior. The elastic properties of individual phases ${ }^{29}$ and the required properties for the constitutive model for the matrix ${ }^{30}$ are also 
shown in Table 2. The GMC results are compared with the FEA analysis to evaluate GMC as a homogenization tool for studying the multi-phased structures which would pave the way for enabling the multi-scale modeling for analyzing Ni-based superalloy engine components.

Table 2. Nickel-Bases Super alloy (CMSX-4), elastic properties for Matrix and Inclusion ${ }^{29}$ and Plastic properties for the Matrix ${ }^{30}$

\begin{tabular}{|c|c|c|c|c|c|c|c|c|}
\hline & $E(G P a)$ & $\boldsymbol{\vartheta}$ & $G(\mathbf{G P a})$ & $\boldsymbol{n}$ & $\dot{\boldsymbol{a}}$ & $\boldsymbol{h}_{\mathbf{0}}(\mathrm{MPa})$ & $\boldsymbol{\tau}_{\mathbf{0}}(\mathrm{MPa})$ & $\boldsymbol{\tau}_{\boldsymbol{s}}(\mathrm{MPa})$ \\
\hline Matrix $(\boldsymbol{\gamma})$ & 129 & 0.391 & 137 & 10 & 0.001 & 350 & 260 & 775 \\
\hline Inclusion $\left(\boldsymbol{\gamma}^{\prime}\right)$ & 114 & 0.405 & 130 & -- & -- & -- & -- & -- \\
\hline
\end{tabular}

\section{A. Numerical implementation using FEA}

Uniaxial loading with a $1 \mathrm{GPa}$ load applied in the z-direction was considered. Periodic boundary conditions were applied in all the three directions. The geometry of the modeled two-phased single crystal (CMSX-4) is illustrated in Figure 2. Three different finite element meshes were considered for studying the convergence of the solution.

a)

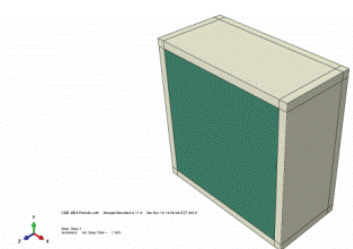

b)

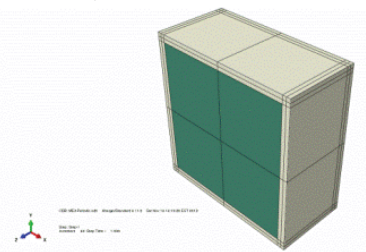

c)

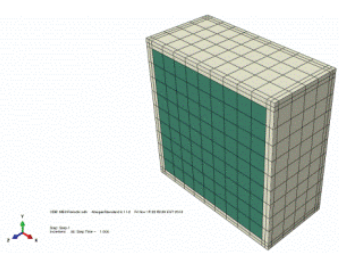

Figure 2. Three different mesh refinements for studying the multi-phased single crystal. The sample is cut in half in the z-direction to show the inclusion. a) one element each in matrix thickness and inclusion thickness direction (named as (111)) b) two elements each in matrix and inclusion thickness directions (named as (222)), and c) two elements in matrix thickness direction, and 9 elements in Inclusion thickness direction (named as (292))

Figure 2a shows a mesh containing one element in the matrix in the thickness direction while having one element for inclusion. This case is named as (111). The second case with two elements in each of the matrix and inclusion thickness direction, case (222), is illustrated in Figure 2b. In the third case, the number of elements in the inclusion thickness direction is increased to nine (292) (Figure 2c).

First, the convergence of the FEA solution was studied. The average axial stress-strain curve of the samples is shown in Figure 3. It is observed that all the studied cases demonstrate very good agreement for both the matrix and inclusion, and as a result for the entire domain. In addition, in Figure 4 the axial stress distributions on the studied cross-section demonstrate close match. The case (111) showed uniform distribution for each component through the studied section, while case (292) captured the varitation; however, they are negligible relative to the average field distribution values. It should be noted that the stress values are maximum in the corners of the inclusion region which can only be observed in case (292). Therefore, for the application that requires very high accuracy in the field solution, the models containing higher number of elements should be utilized.

a)

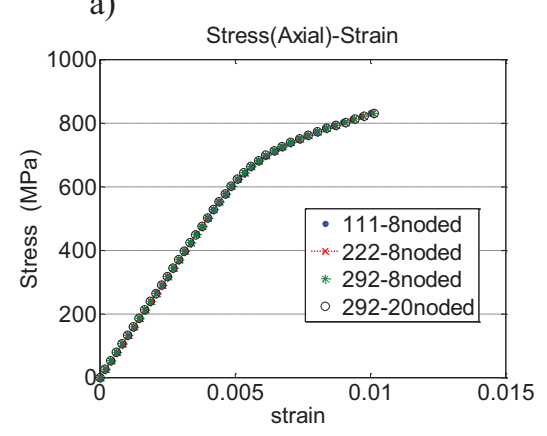

b)

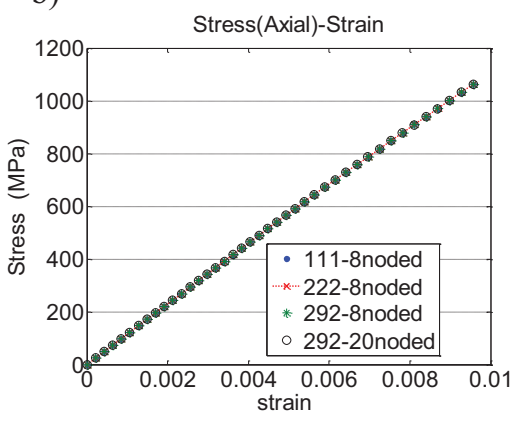

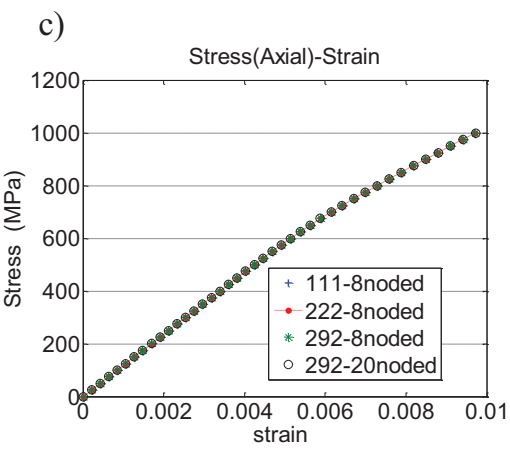

Figure 3. Axial stress vs. Axial strain for the studied multi-phased single crystal for cases 111, 222, 292 in a) only Matrix b) only Inclusion c) whole domain 
a)

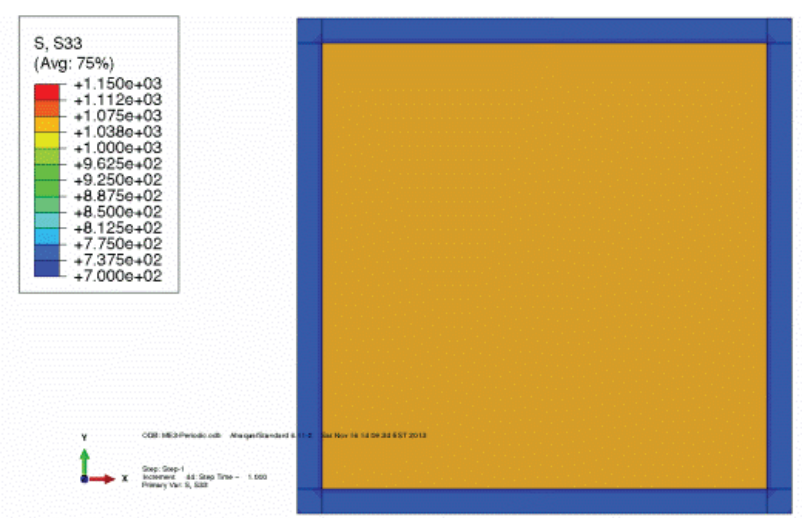

b)

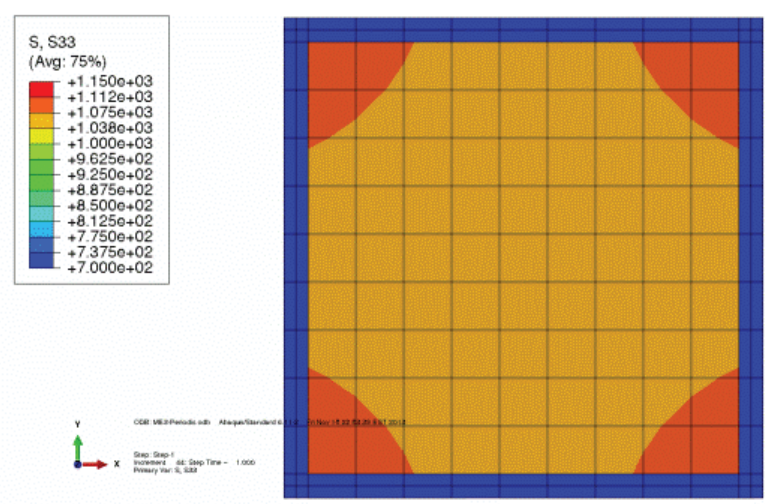

Figure 4. Axial stress distribution (MPa) on the cut plane for a) (111) case b) (292) case

\section{B. Numerical Implementation using GMC}

Same Nickel-based super alloy (CMSX4) sample studied in the previous section is modeled using GMC based on the (111) mesh and (292) mesh. The results for the average axial stress-axial strain and the vonMisses stress-time responses, and vonMisses stress distributions on the chosen cross-section are shown in Figures 5 and 6.

a)

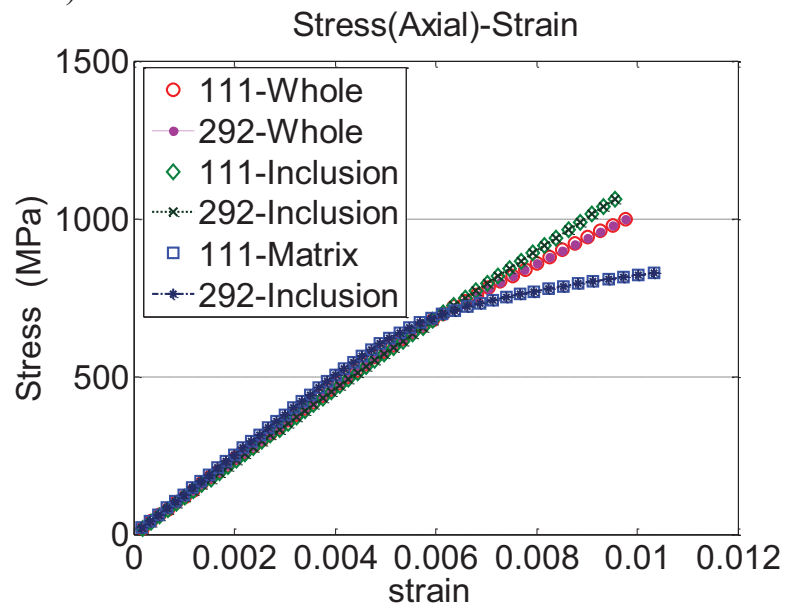

b)

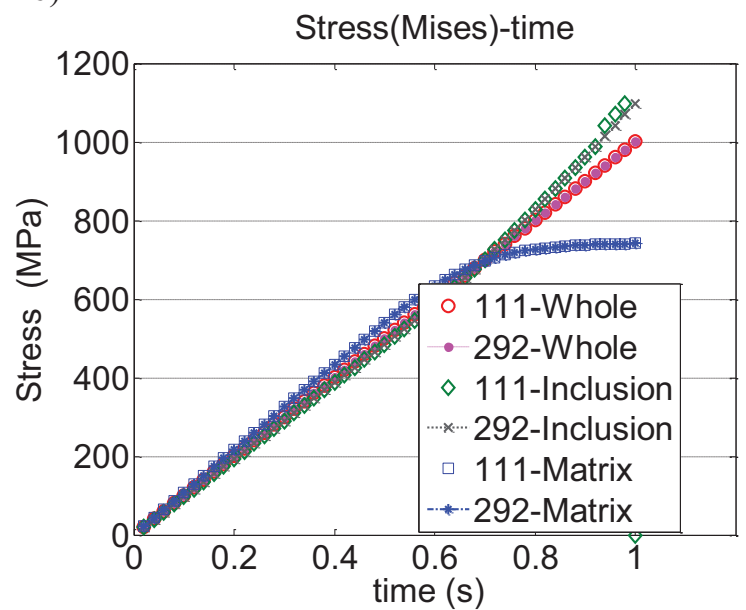

Figure 5. Behavior of the studied multi-phased single crystal in GMC for cases 111 and 292 for whole domain, only inclusion and only matrix a) Axial stress vs. Axial strain b) vonMisses stress vs. time 
From Figures 5 and 6, no difference is observed in the reported results from the cases (111) and (292). This was expected as the GMC method is a homogenization scheme, demonstrating the average value in the phase.
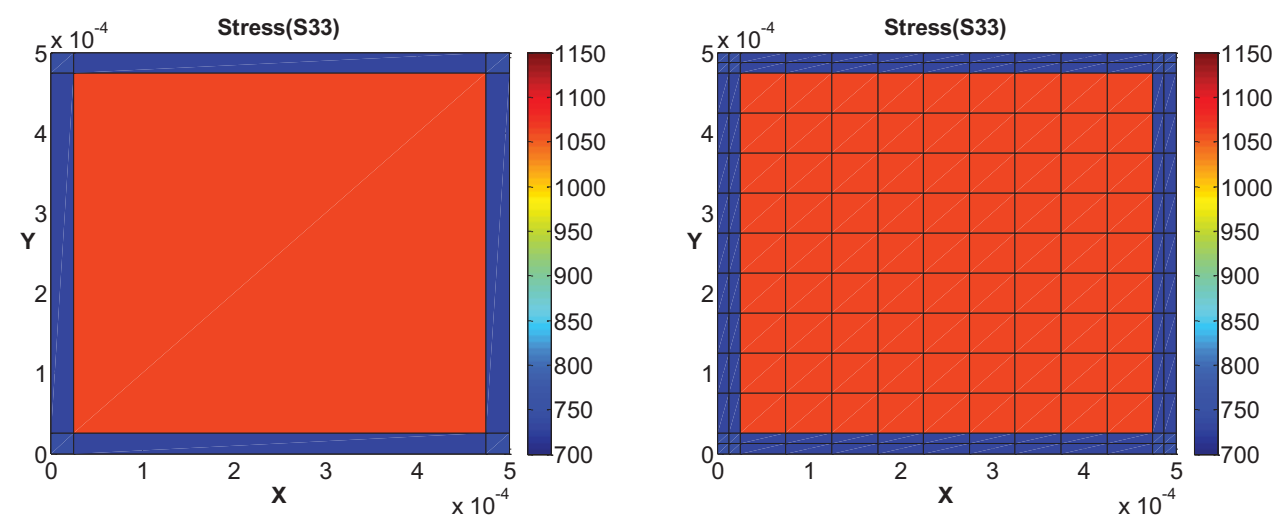

Figure 6. Results from GMC for cases 111 (Left) and 292 (Right) on same cut planes as shown in Fig. 5.2 for Axial stress distribution $(\mathrm{MPa})$

\section{FEA and GMC comparison}

The reported results from FEA and GMC are compared in this section to evaluate the credibility of GMC for studying the two-phased single crystal s like Nickel-based super allows. The results showing the axial stress against the axial strain and the vonMisses stress in time curves obtained from GMC and FEA (shown in Figure 7) demonstrate excellent agreement for both the inclusion and the matrix phases as well as the entire domain.

a)

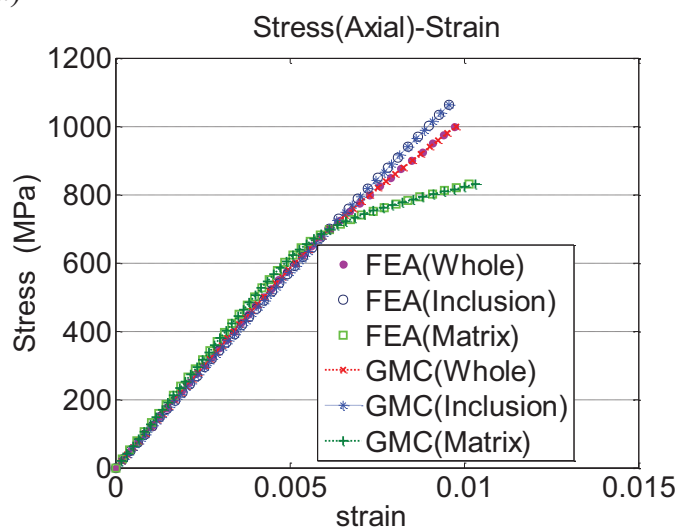

b)

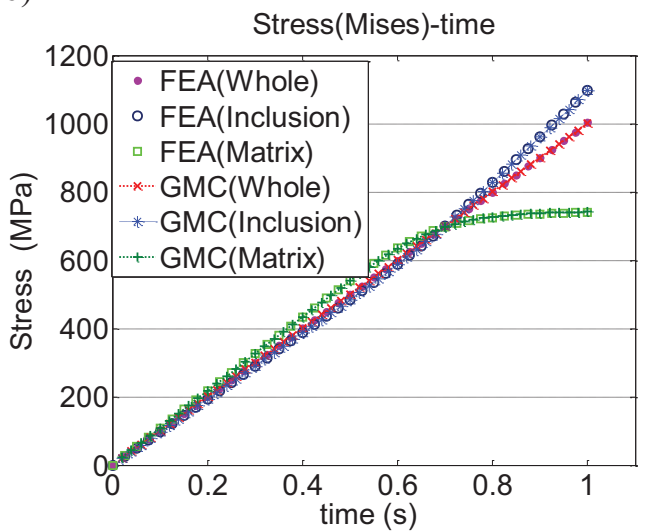

Figure 7. Results from comparison of GMC and FEA for whole domain, only inclusion and only matrix a) Axial stress vs. Axial strain b) vonMisses stress vs. time

The distributions of the individual components on the chosen cross-section are compared in Figure 8. For both GMC and FEA, the simplest case of (111) is chosen for the comparison. Based on the excellent agreement obtained, it can be concluded that the GMC is a reliable method for studying the two-phased single crystal materials. Although the simple geometry of this type of material allows modeling the CMSX-4 unit cell using the least required number of elements in FEA and GMC, still there is a significant advantage (63.7x) in the computational cost of GMC. 
a)
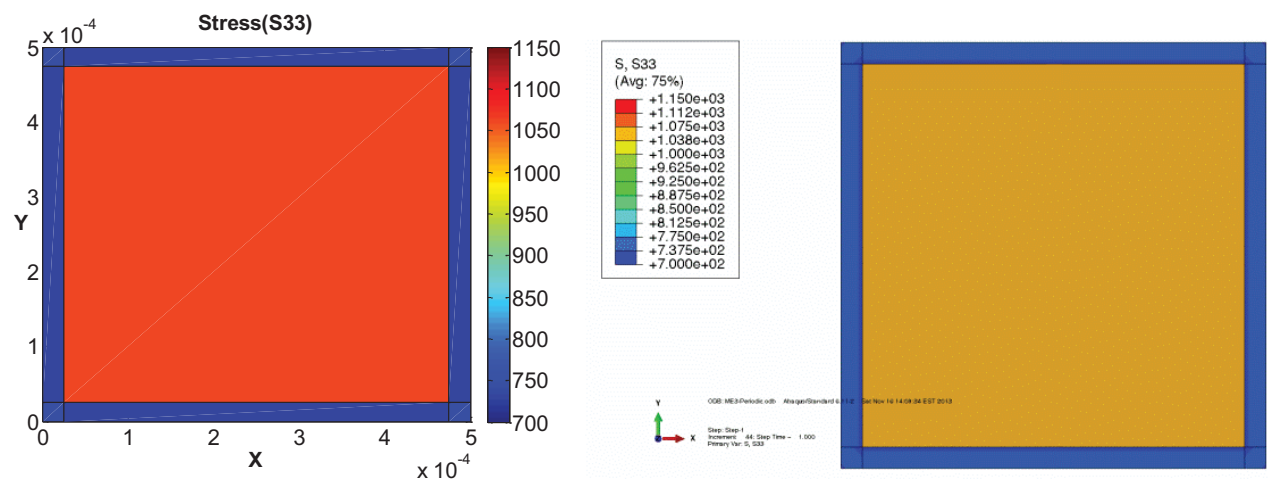

b)
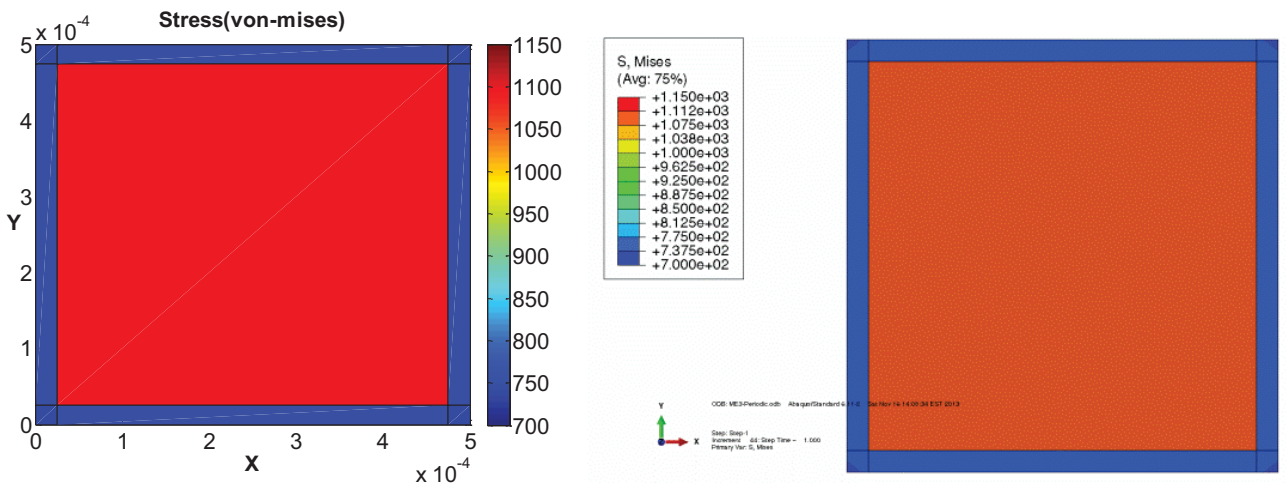

c)
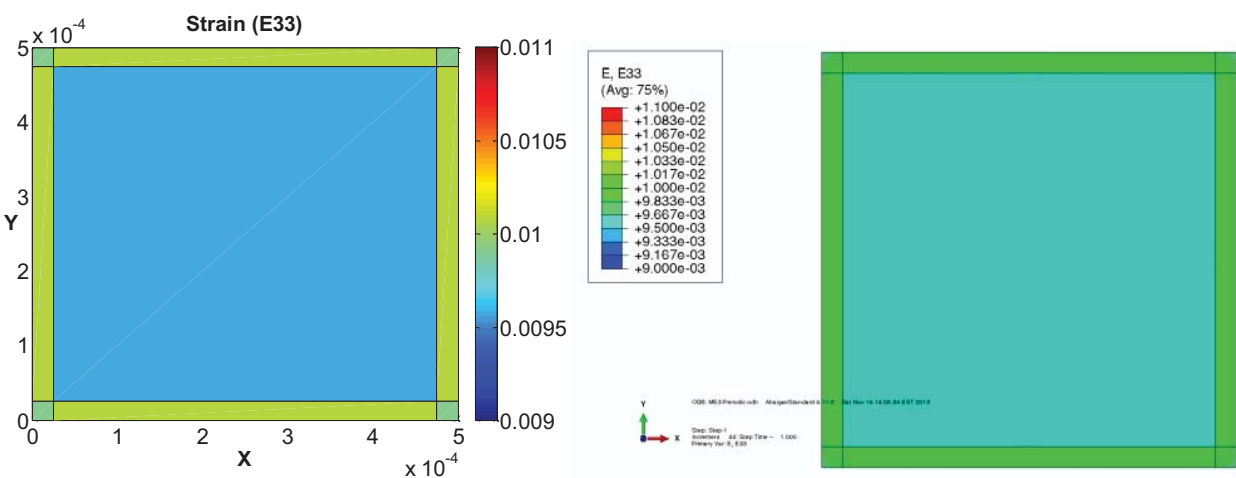

Figure 8. Results from comparison of GMC (Left) and FEA (Right) for case 111 on the cut planes as shown in Fig. 30 for a) Axial stress distribution (MPa) b) Axial strain (lower bound is 0.009 and upper bound is 0.011) c) vonMisses stress distribution (MPa)

\section{Multi-Scale Simulation}

In order to implement a multi-scale analysis on realistic size problems, a macro-oscale FEA model is linked to the GMC micromechanics. The FEA determines the stress/strain on the integration points of the elements which is updated in each time step. These localized quantities are applied to the RUCs through GMC allowing the homogenization process in RUC. For the purpose of demonstrating this functionality, a segment of a real-size turbine disk is analyzed. The disk is $254 \mathrm{~mm}$ long and is exposed to a centrifugal load $\left(\rho \omega^{2}\right)$ with the magnitude of $25.5 \frac{\mathrm{kg} \cdot \mathrm{rad}^{2}}{\mathrm{~mm}^{3} \cdot \mathrm{s}^{2}}$ (corresponding to the squared angular speed of $3 \mathrm{e} 9\left(\frac{\mathrm{rad}}{\mathrm{sec}}\right)^{2}$ ). Considering the symmetry of the geometry and the load, 1/8 of the disk is analyzed applying appropriate symmetric boundary conditions. It is important to note that the symmetric assumption is valid strictly only at the macro-scale. The two-phased CMSX-4 superalloy was used as the material model for each element in the micro-scale domain. 
a)

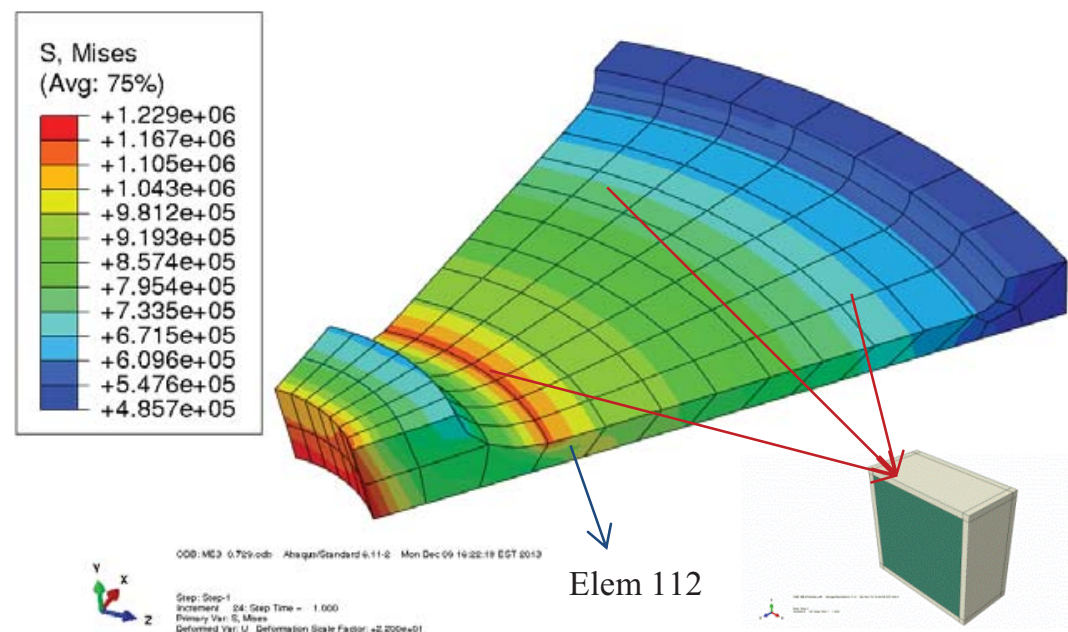

c)

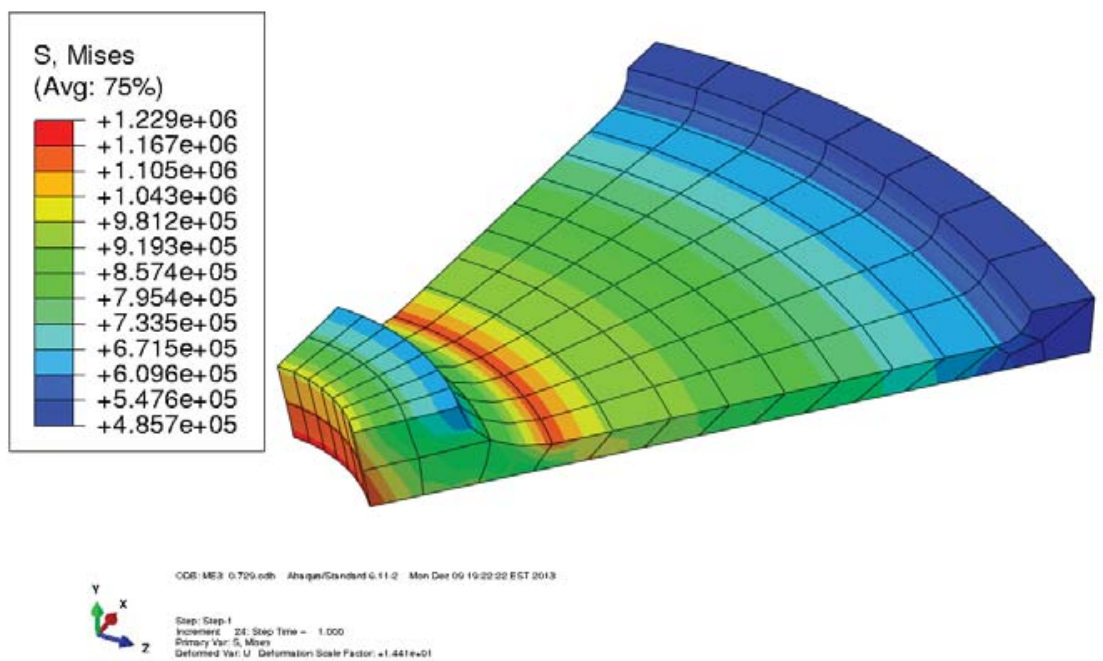

b)
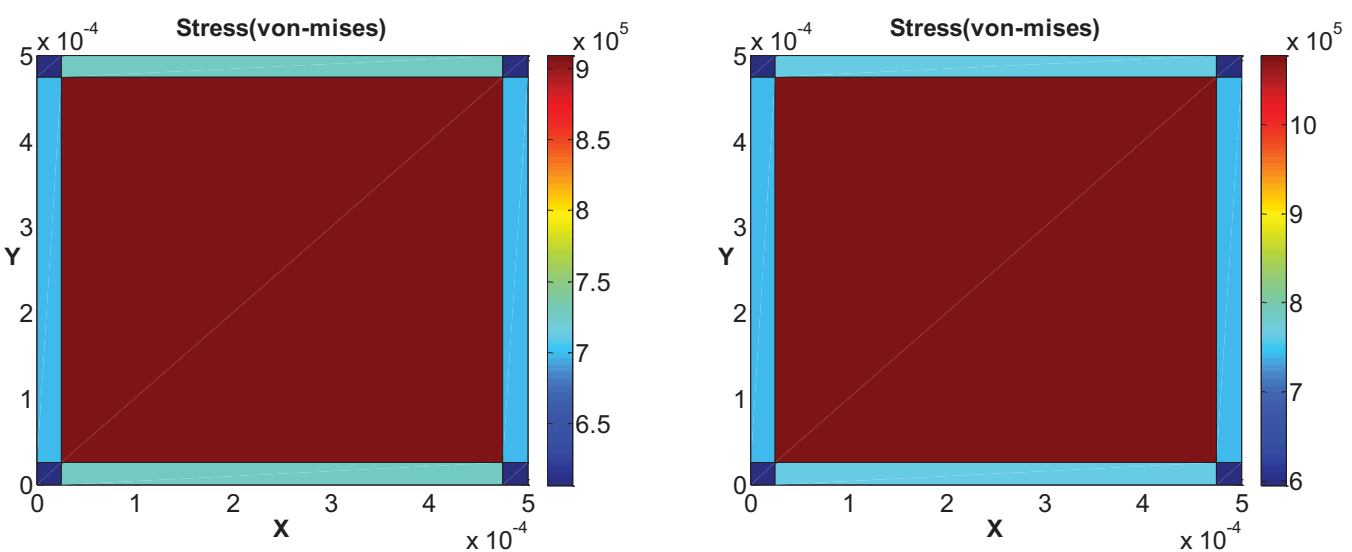

Figure 9. vonMisses stress distribution $\left(\times 10^{-3} \mathrm{MPa}\right)$ in the real-size disk example a) linking macro-scale to

micro-scale using FEA/GMC b) applying the standard FEA model with a macro-scale constitutive relationship c)

Micro-level study at two integration points on a chosen cross-section (element number 112)

9

American Institute of Aeronautics and Astronautics 
Figure. 9a shows the vonMisses stress distribution in the disk. To compare the macro-scale stress distribution, the vonMisses stress distribution obtained by using a standard macro-scale plasticity model is shown in Figure 9b. In the standard FEA model, the global stress-strain curve obtained from the same two-phased CMSX-4 under periodic boundary condition was used as the constitutive model. It is important to note that the stress/strain variation per element in the standard FEA follows the element interpolation while in the multi-scale model the GMC can evaluate the stress/strain distribution at the micro-scale consistent with the interpolated macro-scale values of the macro-scale finite element.

The vonMisses stress distributions shown in Figure $9 \mathrm{a}$ and $9 \mathrm{~b}$ demonstrate that the multi-scale linking. using GMC homogenization under finite element framework. predicts expected distribution at macro-scale. The vonMisses stress distributions on a chosen cross-section halfway through the depth of the unitcell for the two of the integration points of element 112 (shown in Figure 9a) are demonstrated in Figure 9c. The results show that the local field quantity distributions at the grain-level in a real-sized model can be determined using the developed multi-scale model which is extremely difficult with a standard FEA analysis alone due to exorbitant computational cost.

\section{Conclusion}

A multi-scale computational model (employing FEA and GMC) that can capture the microstructural scale stress field for real sized problems, employing crystal plasticity constitutive relations in conjunction with the generalized method of cells (GMC) for homogenization of local field quantities, was developed. Two-phased materials (e.g. ME3) were studied by explicitly defining each individual phases. To this end, the micro-scale behavior of the twophased materials were determined using GMC and compared to that obtained from standard FEA. The GMC analysis achieved high savings in computational cost at a minimal expense of accuracy in the components of local tensor field quantities. GMC showed excellent agreement with the FEA results in terms of the average stress-strain behavior and the field quantity distributions. Finally, the multi-scale aspect of the model, linking the microstructure with the two explicit phases to the macro-scale model, was demonstrated by implementing GMC as a homogenization tool on FEA platform, and investigating a real life turbine engine disc problem. The macro-scale results demonstrated similar distribution when compared to the FEA based analysis thereby verifying the method. In addition, it was shown that the micro-scale distribution of field quantities like vonMisses stress can be extracted on the cross-section of an arbitrarily selected element. The developed multi-scale model may allow engineers to tailor the microstructure for different structural applications.

\section{Acknowledgments}

The first authors would like to thank NASA Glenn Research Center and Ohio Aerospace Institute for providing a summer research fellowship grant to support this work.

\section{References}

${ }^{1}$ Reed, R.C. , The Superalloys: Fundamentals and Applications, Cambridge University Press, Cambridge, 2006.

2 Pollock, T.M., Tin, S., "Nickel-Based Superalloys for Advanced Turbine Engines: Chemistry, Microstructure and Properties," Journal of Propulsion and Power, Vol. 22, No. 2, 2006, pp. 361-374.

${ }^{3}$ Keshavarz, Sh., Ghosh, S., "Multi-scale crystal plasticity finite element model approach to modeling nickel-based superalloys", Acta Materialia, Vol. 61, 2013,pp. 6549-6561.

${ }^{4}$ Huang, Y., "A user-material subroutine incorporating single crystal plasticity in the abaqus finite element program Mech Report 178”, Division of Engineering and Applied Sciences, Harvard University, Cambridge, Massachusetts, 1991.

${ }^{5}$ Kysar, J. W., "Addendum to 'A user-material subroutine incorporating single crystal plasticity in the abaqus finite element program Mech Report 178”, Division of Engineering and Applied Sciences, Harvard University, Cambridge, Massachusetts, 1999.

${ }^{6}$ Ohashi, T., Hidaga, K., Saito, M., "Quantitative study of the plastic slip deformation and formation of internal stresses in Ni-base superalloys", Materials Science \& Engineering A (Structural Materials: Properties, Microstructure and Processing), Vol. 238, 1997, pp. 42-49.

${ }^{7}$ MacLachlan, D. W., Gunturi G. S. K., Knowles, D. M., "Modelling the uniaxial creep anisotropy of nickel base single crystal superalloys CMSX-4”, Comput. Mater. Sci, Vol. 25, 2002, pp.129-41

${ }^{8}$ Daniel, R., Tinga, T., Henderson, M. B., "Deformation modelling of the single crystal superalloy CM186LC", Proc. Materials for Advanced Power Engineering (J"ulich) ed J Lecomte-Beckers et al (J"ulich, Germany: Forschungszentrum J"ulich)

${ }^{9}$ Fedelich, B., "A microstructure based constitutive model for the mechanical behavior at high temperatures of nickel-base single crystal superalloys", Comput. Mater. Sci., Vol. 16, 1999, pp. 248-58 .

${ }^{10}$ Fedelich B., "A microstructural model for the monotonic and the cyclic mechanical behavior of single crystals of superalloys at high temperatures”, Int. J. Plast., Vol. 18, 2002, pp. 1-49. 
${ }^{11}$ Busso, E. P., McClintock, F. A., “A dislocation mechanics-based crystallographic model of a B2-type intermetallic alloy”, Int. J. Plast. Vol. 12, 1996, pp.1-28

${ }^{12}$ Busso E P,Meissonnier F T and O’Dowd N P "Gradient-dependent visco-plastic deformation of two-phase single crystals" J. Mech. Phys. Solids, Vol. 48, 2000, 2333-61

${ }^{13}$ Nouailhas, D., Cailletaud, G., 1996. "Finite element analysis of the mechanical behavior of two-phase singlecrystal superalloys", Scripta Materialia, Vol. 34, 1996, pp. 565-571.

${ }^{14}$ Shenoy, M.M., Gordon, A.P., McDowell, D.L., Neu, R.W., "Thermomechanical fatigue behavior of a directionally solidified Ni-base superalloy", Transactions of the ASME, Journal of Engineering Materials and Technology, ASME, USA, Vol. 127,2005 , pp. 325-337.

${ }^{15}$ Aboudi, J., "Mechanics of composite materials" - a unified micromechanical approach. Elsevier, Amsterdam, 1991.

${ }^{16}$ Aboudi, J., "Micromechanical analysis of thermo-inelastic multiphase short-fiber composutes", Composites Engineering, Vol. 5, 1995, pp. 839-850.

${ }^{17}$ Aboudi, J, Pindera, M.J., Arnold, SM., "Higher-order theory for periodic multiphase materials with inelastic phases, Int. J. Plasticity. Vol. 19, 1995, pp. 805-47.

${ }^{18}$ Bednarcyk BA, Pindera M-J., "Inelastic response of a woven carbon/copper composite. Part 2: micromechanics model", J. Compos. Mater., Vol. 34, 2000, pp. 299-331.

${ }^{19}$ Pindera M-J, Bednarcyk A. B., "An efficient implementation of the GMC micromechanics model for multi-phased materials with complex microstructures", Composites B (Eng), Vol. 30, 1999, pp. 87-105.

20 Bednarcyk, B.A. Arnold, S.M., MAC/GMC 4.0 User's Manual Volume 2: Keywords Manual NASA Technical Memorandum 212077, Vol. 2, 2002a.

21 Bednarcyk, B.A., Arnold, S.M., MAC/GMC 4.0 User's Manual Volume 3: Example Manual. NASA Technical Memorandum 212077, Vol. 3, 2002b.

${ }^{22}$ Taylor, G. I., "Plastic strain in Metals", Journal of the Institute of Metals. Vol. 62, 1938, pp. 307.

${ }^{23}$ Hill, R.,. "Ceneralized constitutive relations for incremental deformation of metal crystals by multislip", Journal of Mechanics and Physics of Solids", Vol. 14, 1966, pp. 95.

${ }^{24}$ Hill, R. and Rice, J. R., "Constitutive analysis of elastic-plastic crystals at arbitrary strain", Journal of Mechanics and Physics of Solids, Vol. 15, 1972, pp. 401.

${ }^{25}$ Peirce, D., Asaro, R. J., Needleman, A., “An Analysis of Nonuniform and Localized Deformation in Ductile Single Crystals", Acta. Metall, Vol. 30, 1982, pp. 1087.

${ }^{26}$ Asaro, R., "Micromechanics of Crystals and Polycrystals", Adv. Appl. Mech, Vol. 23, 1983a., pp. 1.

${ }^{27}$ Abaqus Users Manual, Version 6.8, Simulia Inc.

${ }^{28}$ Bednarcyk, B.A, Pindera, M-J., "Inelastic response of a woven carbon/copper composite. Part 2: micromechanics model", J. Compos. Mater., Vol. 34, No. 4, 1983a. pp. 299-331.

${ }^{29}$ Siebo" rger, D., Knake, H., Glatzel, U., "Temperature dependence of the elastic moduli of the nickel-base superalloy CMSX-4 and its isolated phases", Materials Science and Engineering A, Vol. 298, 2001, pp. $26-33$.

${ }^{30}$ Eidel, B., "Crystal plasticity finite-element analysis versus experimental results of pyramidal indentation into (001) fcc single crystal", Acta Materialia, Vol. 59, 2011, pp. 1761-1771. 